\title{
Profinite groups with restricted centralizers of $\pi$-elements
}

\author{
Cristina Acciarri and Pavel Shumyatsky
}

\begin{abstract}
A group $G$ is said to have restricted centralizers if for each $g$ in $G$ the centralizer $C_{G}(g)$ either is finite or has finite index in $G$. Shalev showed that a profinite group with restricted centralizers is virtually abelian. Given a set of primes $\pi$, we take interest in profinite groups with restricted centralizers of $\pi$-elements. It is shown that such a profinite group has an open subgroup of the form $P \times Q$, where $P$ is an abelian pro- $\pi$ subgroup and $Q$ is a pro- $\pi^{\prime}$ subgroup. This significantly strengthens a result from our earlier paper.
\end{abstract}

\section{Introduction}

A group $G$ is said to have restricted centralizers if for each $g$ in $G$ the centralizer $C_{G}(g)$ either is finite or has finite index in $G$. This notion was introduced by Shalev in [13] where he showed that a profinite group with restricted centralizers is virtually abelian. We say that a profinite group has a property virtually if it has an open subgroup with that property. The article [3] handles profinite groups with restricted centralizers of $w$-values for a multilinear commutator word $w$. The theorem proved in [3] says that if $w$ is a multilinear commutator word and $G$ is a profinite group in which the centralizer of any $w$-value is either finite or open, then the verbal subgroup $w(G)$ is virtually abelian. In [1] we study profinite groups in which $p$-elements have restricted centralizers, that is, groups in which $C_{G}(x)$ is either finite or open for any $p$-element $x$. The following theorem was proved.

2010 Mathematics Subject Classification. 20E18, 20F24.

Key words and phrases. Profinite groups, centralizers, $\pi$-elements, FC-groups.

This research was supported by the Conselho Nacional de Desenvolvimento Científico e Tecnológico $(\mathrm{CNPq})$, and Fundação de Apoio à Pesquisa do Distrito Federal (FAPDF), Brazil. 
THEOREM 1.1. Let $p$ be a prime and $G$ a profinite group in which the centralizer of each p-element is either finite or open. Then $G$ has a normal abelian pro-p subgroup $N$ such that $G / N$ is virtually pro-p' .

The present paper grew out of our desire to determine whether this result can be extended to profinite groups in which the centralizer of each $\pi$-element, where $\pi$ is a fixed set of primes, is either finite or open. As usual, we say that an element $x$ of a profinite group $G$ is a $\pi$-element if the order of the image of $x$ in every finite continuous homomorphic image of $G$ is divisible only by primes in $\pi$ (see [10, Section 2.3] for a formal definition of the order of a profinite group).

It turned out that the techniques used in the proof of Theorem 1.1 were not quite adequate for handling the case of $\pi$-elements. The basic difficulty stems from the fact that (pro)finite groups in general do not possess Hall $\pi$-subgroups.

In the present paper we develop some new techniques and establish the following theorem about finite groups.

If $\pi$ is a set of primes and $G$ a finite group, write $O^{\pi^{\prime}}(G)$ for the unique smallest normal subgroup $M$ of $G$ such that $G / M$ is a $\pi^{\prime}$-group. The conjugacy class containing an element $g \in G$ is denoted by $g^{G}$.

TheOREM 1.2. Let $n$ be a positive integer, $\pi$ be a set of primes, and $G$ a finite group such that $\left|g^{G}\right| \leq n$ for each $\pi$-element $g \in G$. Let $H=O^{\pi^{\prime}}(G)$. Then $G$ has a normal subgroup $N$ such that

(1) The index $[G: N]$ is $n$-bounded;

(2) $[H, N]=[H, H]$;

(3) The order of $[H, N]$ is n-bounded.

Throughout the article we use the expression " $(a, b, \ldots)$-bounded" to mean that a quantity is finite and bounded by a certain number depending only on the parameters $a, b, \ldots$

The proof of Theorem 1.2 uses some new results related to Neumann's BFC-theorem [8]. In particular, an important role in the proof is played by a recent probabilistic result from [2]. Theorem 1.2 provides a highly effective tool for handling profinite groups with restricted centralizers of $\pi$-elements. Surprisingly, the obtained result is much stronger than Theorem 1.1 even in the case where $\pi$ consists of a single prime.

THEOREM 1.3. Let $\pi$ be a set of primes and $G$ a profinite group in which the centralizer of each $\pi$-element is either finite or open. Then $G$ has an open subgroup of the form $P \times Q$, where $P$ is an abelian pro- $\pi$ subgroup and $Q$ is a pro- $\pi^{\prime}$ subgroup. 
Thus, the improvement over Theorem 1.1 is twofold - the result now covers the case of $\pi$-elements and provides additional details clarifying the structure of groups in question. Furthermore, it is easy to see that Theorem 1.3 extends Shalev's result [13] which can be recovered by considering the case where $\pi=\pi(G)$ is the set of all prime divisors of the order of $G$.

We now have several results showing that if the elements of a certain subset $X$ of a profinite group $G$ have restricted centralizers, then the structure of $G$ is very special. This suggests the general line of research whose aim would be to determine which subsets of $G$ have the above property. At present we are not able to provide any insight on the problem. Perhaps one might start with the following question:

Let $n$ be a positive integer. What can be said about a profinite group $G$ such that if $x \in G$ then $C_{G}\left(x^{n}\right)$ is either finite or open?

Proofs of Theorems 1.2 and 1.3 will be given in Sections 2 and 3, respectively.

\section{Proof of Theorem 1.2}

The following lemma is taken from [1]. If $X \subseteq G$ is a subset of a group $G$, we write $\langle X\rangle$ for the subgroup generated by $X$ and $\left\langle X^{G}\right\rangle$ for the minimal normal subgroup of $G$ containing $X$.

LEMMA 2.1. Let $i, j$ be positive integers and $G$ a group having a subgroup $K$ such that $\left|x^{G}\right| \leq i$ for each $x \in K$. Suppose that $|K| \leq j$. Then $\left\langle K^{G}\right\rangle$ has finite $(i, j)$-bounded order.

If $K$ is a subgroup of a finite group $G$, we denote by

$$
\operatorname{Pr}(K, G)=\frac{|\{(x, y) \in K \times G:[x, y]=1\}|}{|K||G|}
$$

the relative commutativity degree of $K$ in $G$, that is, the probability that a random element of $G$ commutes with a random element of $K$. Note that

$$
\operatorname{Pr}(K, G)=\frac{\sum_{x \in K}\left|C_{G}(x)\right|}{|K||G|}
$$

It follows that if $\left|x^{G}\right| \leq n$ for each $x \in K$, then $\operatorname{Pr}(K, G) \geq \frac{1}{n}$.

The next result was obtained in [2, Proposition 1.2]. In the case where $K=G$ this is a well known theorem, due to P. M. Neumann [9].

Proposition 2.2. Let $\epsilon>0$, and let $G$ be a finite group having a subgroup $K$ such that $\operatorname{Pr}(K, G) \geq \epsilon$. Then there is a normal subgroup $T \leq G$ and a subgroup $B \leq K$ such that the indexes $[G: T]$ and $[K: B]$, and the order of the commutator subgroup $[T, B]$ are $\epsilon$-bounded. 
We will now embark on the proof of Theorem 1.2 .

Assume the hypothesis of Theorem 1.2. Let $X$ be the set of all $\pi$-elements of $G$. Clearly, $H=\langle X\rangle$. Given an element $g \in H$, we write $l(g)$ for the minimal number $l$ with the property that $g$ can be written as a product of $l$ elements of $X$. The following result is straightforward from [4, Lemma 2.1].

Lemma 2.3. Let $K \leq H$ be a subgroup of index $m$ in $H$, and let $b \in H$. Then the coset $K b$ contains an element $g$ such that $l(g) \leq m-1$.

Let $m$ be the maximum of indices of $C_{H}(x)$ in $H$ where $x \in X$. Obviously, we have $m \leq n$.

Lemma 2.4. For any $x \in X$ the subgroup $[H, x]$ has m-bounded order.

Proof. Take $x \in X$. Since the index of $C_{H}(x)$ in $H$ is at most $m$, by Lemma 2.3, we can choose elements $y_{1}, \ldots, y_{m}$ in $H$ such that $l\left(y_{i}\right) \leq$ $m-1$ and the subgroup $[H, x]$ is generated by the commutators $\left[y_{i}, x\right]$, for $i=1, \ldots, m$. For any such $i$ write $y_{i}=y_{i 1} \ldots y_{i(m-1)}$, with $y_{i j} \in$ $X$. Using standard commutator identities we can rewrite $\left[y_{i}, x\right]$ as a product of conjugates in $H$ of the commutators $\left[y_{i j}, x\right]$. Let $\left\{h_{1}, \ldots, h_{s}\right\}$ be the conjugates in $H$ of all elements from the set $\left\{x, y_{i j} \mid 1 \leq i, j \leq\right.$ $m$ \}. Note that the number $s$ here is $m$-bounded. This follows form the fact that $C_{H}(x)$ has index at most $m$ in $H$ for each $x \in X$. Put $T=\left\langle h_{1}, \ldots, h_{s}\right\rangle$. Since $[H, x]$ is contained in the commutator subgroup $T^{\prime}$, it is sufficient to show that $T^{\prime}$ has $m$-bounded order. Observe that the centre $Z(T)$ has index at most $m^{s}$ in $T$, since the index of $C_{H}\left(h_{i}\right)$ is at most $m$ in $H$ for any $i=1, \ldots, s$. Thus, by Schur's theorem [11, 10.1.4], we conclude that the order of $T^{\prime}$ is $m$-bounded, as desired.

Select $a \in X$ such that $\left|a^{H}\right|=m$. Choose $b_{1}, \ldots, b_{m}$ in $H$ such that $l\left(b_{i}\right) \leq m-1$ and $a^{H}=\left\{a^{b_{i}} ; i=1, \ldots, m\right\}$. The existence of the elements $b_{i}$ is guaranteed by Lemma 2.3. Set $U=C_{G}\left(\left\langle b_{1}, \ldots, b_{m}\right\rangle\right)$. Note that the index of $U$ in $G$ is $n$-bounded. Indeed, since $l\left(b_{i}\right) \leq m-1$ we can write $b_{i}=b_{i 1} \ldots b_{i(m-1)}$, where $b_{i j} \in X$ and $i=1, \ldots, m$. By the hypothesis the index of $C_{G}\left(b_{i j}\right)$ in $G$ is at most $n$ for any such element $b_{i j}$. Thus, $[G: U] \leq n^{(m-1) m}$.

The next result is somewhat analogous to [14, Lemma 4.5].

Lemma 2.5. If $u \in U$ and $u a \in X$, then $[H, u] \leq[H, a]$.

Proof. Assume that $u \in U$ and $u a \in X$. For each $i=1, \ldots, m$ we have $(u a)^{b_{i}}=u a^{b_{i}}$, since $u$ belongs to $U$. We know that $u a \in X$ so taking into account the hypothesis on the cardinality of the conjugacy 
class of $u a$ in $H$, we deduce that $(u a)^{H}$ consists exactly of the elements $u a^{b_{i}}$, for $i=1, \ldots, m$. Thus, given an arbitrary element $h \in H$, there exists $b \in\left\{b_{1}, \ldots, b_{m}\right\}$ such that $(u a)^{h}=u a^{b}$ and so $u^{h} a^{h}=u a^{b}$. It follows that $[u, h]=a^{b} a^{-h} \in[H, a]$, and the result holds.

LEMma 2.6. The order of the commutator subgroup of $H$ is $n$ bounded.

Proof. Let $U_{0}$ be the maximal normal subgroup of $G$ contained in $U$. Recall that, by the remark made before Lemma 2.5, $U$ has $n$ bounded index in $G$. It follows that the index $\left[G: U_{0}\right]$ is $n$-bounded as well.

By the hypothesis $a$ has at most $n$ conjugates in $G$, say $\left\{a^{g_{1}}, \ldots, a^{g_{n}}\right\}$. Let $T$ be the normal closure in $G$ of the subgroup $[H, a]$. Note that the subgroups $\left[H, a^{g_{i}}\right]$ are normal in $H$, therefore $T=\left[H, a^{g_{1}}\right] \ldots\left[H, a^{g_{n}}\right]$. By Lemma 2.4 each of the subgroups $\left[H, a^{g_{i}}\right]$ has $n$-bounded order. We conclude that the order of $T$ is $n$-bounded.

Let $Y=X a^{-1} \cap U$. Note that for any $y \in Y$ the product ya belongs to $X$. Therefore, by Lemma 2.5. for any $y \in Y$, the subgroup $[H, y]$ is contained in $[H, a]$. Thus,

$$
[H, Y] \leq T .
$$

Observe that for any $u \in U_{0}$ the commutator $\left[u, a^{-1}\right]=a^{u} a^{-1}$ lies in $Y$ and so

$$
\left[H,\left[U_{0}, a^{-1}\right]\right] \leq[H, Y] .
$$

Since $\left[U_{0}, a^{-1}\right]=\left[U_{0}, a\right]$, we deduce from (1) and (2) that

$$
\left[H,\left[U_{0}, a\right]\right] \leq T \text {. }
$$

Since $T$ has $n$-bounded order, it is sufficient to show that the derived group of the quotient $H / T$ has finite $n$-bounded order. We pass now to the quotient $G / T$ and for the sake of simplicity the images of $G$, $H, U, U_{0}, X$ and $Y$ will be denoted by the same symbols. Note that by (1) the set $Y$ becomes central in $H$ modulo $T$. Containment (3) shows that $\left[U_{0}, a\right] \leq Z(H)$. This implies that if $b \in U_{0}$ is a $\pi$-element, then $[b, a] \in Z(H)$ and the subgroup $\langle a, b\rangle$ is nilpotent. Thus the product $b a$ is a $\pi$-element too and so $b \in Y$. Hence, all $\pi$-elements of $U_{0}$ are contained in $Y$ and, in view of (1), we deduce that they are contained in $Z(H)$.

Next we consider the quotient $G / Z(H)$. Since the image of $U_{0}$ in $G / Z(H)$ is a $\pi^{\prime}$-group and has $n$-bounded index in $G$, we deduce that the order of any $\pi$-subgroup in $G / Z(H)$ is $n$-bounded. In particular, there is an $n$-bounded constant $C$ such that for every $p \in \pi$ the order of the Sylow $p$-subgroup of $G / Z(H)$ is at most $C$. Because of Lemma 
2.1 for any $p \in \pi$ each Sylow $p$-subgroup of $G / Z(H)$ is contained in a normal subgroup of $n$-bounded order. We deduce that all such Sylow subgroups of $G / Z(H)$ are contained in a normal subgroup of $n$-bounded order. Since $H$ is generated by $\pi$-elements, it follows that the order of $H / Z(H)$ is $n$-bounded. Thus, in view of Schur's theorem [11, 10.1.4], we conclude that $\left|H^{\prime}\right|$ is $n$-bounded, as desired.

We will now complete the proof of Theorem 1.2 .

Proof. Assume first that $H$ is abelian. In this case the set $X$ of $\pi$-elements is a subgroup, that is, $X=H$. By the hypothesis we have $\left|x^{G}\right| \leq n$ for any element $x \in H$ and so the relative commutativity degree $\operatorname{Pr}(H, G)$ of $H$ in $G$ is at least $\frac{1}{n}$. Thus, by virtue of Proposition 2.2, there is a normal subgroup $T \leq G$ and a subgroup $B \leq H$ such that the indexes $[G: T]$ and $[H: B]$, and the order of the commutator subgroup $[T, B]$ are $n$-bounded.

Since $H$ is a normal $\pi$-subgroup and $[G: H]$ is a $\pi^{\prime}$-number, by the Schur-Zassenhaus Theorem [5, Theorem 6.2.1] the subgroup $H$ admits a complement $L$ in $G$ such that $G=H L$ and $L$ is a $\pi^{\prime}$-subgroup. Set $T_{0}=T \cap L$. Observe that the index $\left[L: T_{0}\right]$ is $n$-bounded since it is at most the index of $T$ in $G$. Thus we deduce that the index of $H T_{0}$ is $n$-bounded in $G$, as well.

We claim that the order of $\left[H, T_{0}\right]$ is $n$-bounded. Indeed, the $\pi^{\prime}$-subgroup $T_{0}$ acts coprimely on the the abelian $\pi$-subgroup $B_{1}=$ $B\left[B, T_{0}\right]$, and so we have $B_{1}=C_{B_{1}}\left(T_{0}\right) \times\left[B_{1}, T_{0}\right]([\mathbf{7}$, Corollary 1.6.5] $)$. Note that $\left[B_{1}, T_{0}\right]=\left[B, T_{0}\right]$. Since the oder of $\left[B, T_{0}\right]$ is $n$-bounded (being at most the order of $[T, B])$, we deduce that the index $\left[B_{1}: C_{B_{1}}\left(T_{0}\right)\right]$ is $n$-bounded. In combination with the fact that $[H: B]$ is $n$-bounded, we obtain that the index $\left[H: C_{B_{1}}\left(T_{0}\right)\right]$ is $n$-bounded and so in particular $\left[H: C_{H}\left(T_{0}\right)\right]$ is $n$-bounded. Since $T_{0}$ acts coprimely on the abelian normal $\pi$-subgroup $H$, we have $H=C_{H}\left(T_{0}\right) \times\left[H, T_{0}\right]$. Thus we obtain that the order of the commutator subgroup $\left[H, T_{0}\right]$ is $n$-bounded, as claimed. Let $T_{1}=C_{T_{0}}\left(\left[H, T_{0}\right]\right)$ and remark that the index $\left[T_{0}: T_{1}\right]$ of $T_{1}$ in $T_{0}$ is $n$-bounded too. Set $N=H T_{1}$. From the fact that the indexes $\left[T_{0}: T_{1}\right]$ and $\left[G: H T_{0}\right]$ are both $n$-bounded, we deduce that the index of $N$ in $G$ is $n$-bounded, as well.

Note that $N$ is normal in $G$ since the image of $N$ in $G / H \cong L$ is isomorphic to $T_{1}$ which is normal in $L$. Furthermore, we have $\left[H, T_{1}, T_{1}\right]=1$, since $T_{1}=C_{T_{0}}\left(\left[H, T_{0}\right]\right)$. Hence by the standard properties of coprime actions we have $\left[H, T_{1}\right]=1$ ([7, Corollary 1.6.4]). Therefore $[H, N]=1$. This proves the theorem in the particular case where $H$ is abelian. 
In the general case, in view of Lemma 2.6, the commutator subgroup $[H, H]$ is of $n$-bounded order. We pass to the quotient $\bar{G}=G /[H, H]$. The above argument shows that $\bar{G}$ has a normal subgroup $\bar{N}$ of $n$ bounded index such that $\bar{H} \leq Z(\bar{N})$. Here $Z(\bar{N})$ stands for the centre of $\bar{N}$. Let $N$ be the inverse image of $\bar{N}$. We have $[H, N]=[H, H]$ and so $N$ has the required properties. The proof is now complete.

\section{Proof of Theorem 1.3}

We will require the following result taken from [1, Lemma 4.1].

LEMMA 3.1. Let $G$ be a locally nilpotent group containing an element with finite centralizer. Suppose that $G$ is residually finite. Then $G$ is finite.

Profinite groups have Sylow $p$-subgroups and satisfy analogues of the Sylow theorems. Prosoluble groups satisfy analogues of the theorems on Hall $\pi$-subgroups. We refer the reader to the corresponding chapters in [10, Ch. 2] and [15, Ch. 2].

Recall that an automorphism $\phi$ of a group $G$ is called fixed-pointfree if $C_{G}(\phi)=1$, that is, the fixed-point subgroup is trivial. It is a well-known corollary of the classification of finite simple groups that if $G$ is a finite group admitting a fixed-point-free automorphism, then $G$ is soluble (see for example [12] for a short proof). A continuous automorphism $\phi$ of a profinite group $G$ is coprime if for any open $\phi$ invariant normal subgroup $N$ of $G$ the order of the automorphism of $G / N$ induced by $\phi$ is coprime to the order of $G / N$. It follows that if a profinite group $G$ admits a coprime fixed-point-free automorphism, then $G$ is prosoluble. This will be used in the proof of Theorem 1.3 .

Proof of Theorem 1.3. Recall that $\pi$ is a set of primes and $G$ is a profinite group in which the centralizer of every $\pi$-element is either finite or open. We wish to show that $G$ has an open subgroup of the form $P \times Q$, where $P$ is an abelian pro- $\pi$ subgroup and $Q$ is a pro- $\pi^{\prime}$ subgroup.

Let $X$ be the set of $\pi$-elements in $G$. Consider first the case where the conjugacy class $x^{G}$ is finite for any $x \in X$. For each integer $i \geq 1$ set

$$
S_{i}=\left\{x \in X ;\left|x^{G}\right| \leq i\right\} .
$$

The sets $S_{i}$ are closed. Thus, we have countably many sets which cover the closed set $X$. By the Baire Category Theorem [6, Theorem 34] at least one of these sets has non-empty interior. It follows that there is a positive integer $k$, an open normal subgroup $M$, and an element $a \in X$ such that all elements in $X \cap a M$ are contained in $S_{k}$. 
Note that $\left\langle a^{G}\right\rangle$ has finite commutator subgroup, which we will denote by $T$. Indeed, the subgroup $\left\langle a^{G}\right\rangle$ is generated by finitely many elements whose centralizer is open. This implies that the centre of $\left\langle a^{G}\right\rangle$ has finite index in $\left\langle a^{G}\right\rangle$, and by Schur's theorem [11, 10.1.4], we conclude that $T$ is finite, as claimed.

Let $x \in X \cap M$. Note that the product $a x$ is not necessarily in $X$. On the other hand, $a x$ is a $\pi$-element modulo $T$. This is because $\left\langle a^{G}\right\rangle$ becomes an abelian normal $\pi$-subgroup modulo $T$ and the image of $a x$ in the quotient $G /\left\langle a^{G}\right\rangle$ is a $\pi$-element. In other words, there are $y \in X \cap a M$ and $t \in T$ such that $a x=t y$. Observe that $t$ has an open centralizer in $G$ since $t \in T$. In fact $\left[G: C_{G}(t)\right] \leq|T|$. From the equality $a x=t y$ deduce that $\left|x^{G}\right| \leq k^{2}|T|$. This happens for any $x \in X \cap M$. Using a routine inverse limit argument in combination with Theorem 1.2 we obtain that $M$ has an open normal subgroup $N$ such that the index $[M: N]$ and the order of $[H, N]$ are finite. Here $H$ stands for the subgroup generated by all $\pi$-elements of $M$. Choose an open normal subgroup $U$ in $G$ such that $U \cap[H, N]=1$. Then $U \cap M$ is an open normal subgroup of the form $P \times Q$, where $P$ is an abelian pro- $\pi$ subgroup and $Q$ is a pro- $\pi^{\prime}$ subgroup. This proves the theorem in the case where all $\pi$-elements of $G$ have open centralizers.

Assume now that $G$ has a $\pi$-element, say $b$, of infinite order. Since the procyclic subgroup $\langle b\rangle$ is contained in the centralizer $C_{G}(b)$, it follows that $C_{G}(b)$ is open in $G$. This implies that all elements of $X \cap C_{G}(b)$ have open centralizers (because they centralize the procyclic subgroup $\langle b\rangle)$. In view of the above $C_{G}(b)$ has an open subgroup of the form $P \times Q$, where $P$ is an abelian pro- $\pi$ subgroup and $Q$ is a pro- $\pi^{\prime}$ subgroup and we are done.

We will therefore assume that $G$ is infinite while all $\pi$-elements of $G$ have finite orders and there is at least one $\pi$-element, say $d$, such that $C_{G}(d)$ is finite. The element $d$ is a product of finitely many $\pi$-elements of prime power order. At least one of these elements must have finite centralizer. So without loss of generality we can assume that $d$ is a $p$-element for a prime $p \in \pi$.

Let $P_{0}$ be a Sylow $p$-subgroup of $G$ containing $d$. Since $P_{0}$ is torsion, we deduce from Zelmanov's theorem [16] that $P_{0}$ is locally nilpotent. The centralizer $C_{G}(d)$ is finite and so in view of Lemma 3.1 the subgroup $P_{0}$ is finite. Choose an open normal pro- $p^{\prime}$ subgroup $L$ such that $L \cap C_{G}(d)=1$. Note that any finite homomorphic image of $L$ admits a coprime fixed-point-free automorphism (induced by the coprime action of $d$ on $L$ ). Hence $L$ is prosoluble. Let $K$ be a Hall $\pi$-subgroup of $L$. Since any element in $K$ has restricted centralizer, Shalev's result [13. shows that $K$ is virtually abelian. We therefore can choose an 
open normal subgroup $J$ in $L$ such that $J \cap K$ is abelian. If $J \cap K$ is finite then $G$ is virtually pro- $\pi^{\prime}$ and we are done. If $J \cap K$ is infinite, then all $\pi$-elements of $J$ have infinite centralizers. This yields that all $\pi$-elements of $J$ have open centralizers in $J$ and in view of the first part of the proof, $J$ has an open normal subgroup of the form $P \times Q$, where $P$ is an abelian pro- $\pi$ subgroup and $Q$ is a pro- $\pi^{\prime}$ subgroup. This establishes the theorem.

\section{References}

[1] C. Acciarri, P. Shumyatsky, A stronger form of Neumann's BFC-theorem, Isr. J. Math. 242, 269-278 (2021). https://doi.org/10.1007/s11856-021-2133-1.

[2] E. Detomi, P. Shumyatsky, On the commuting probability for subgroups of a finite group, Proceedings of the Royal Society of Edinburgh: Section A Mathematics, 1-14 (2021). doi:10.1017/prm.2021.68.

[3] E. Detomi, M. Morigi, P. Shumyatsky, Profinite groups with restricted centralizers of commutators, Proceedings of the Royal Society of Edinburgh, Section A: Mathematics, 150(5) (2020), 2301-2321. doi:10.1017/prm.2019.17.

[4] G. Dierings, P. Shumyatsky, Groups with boundedly finite conjugacy classes of commutators, Quarterly J. Math. 69(3) (2018), 1047-1051.

[5] D. Gorenstein, Finite Groups, Chelsea Publishing Company, New York, 1980.

[6] J. L. Kelley, General topology, Grad. Texts in Math., vol. 27, Springer, New York, 1975.

[7] E. I. Khukhro, Nilpotent groups and their automorphisms, Berlin-New York, de Gruyter, 1993.

[8] B. H. Neumann, Groups covered by permutable subsets, J. London Math. Soc. (3) 29 (1954), 236-248.

[9] P. M. Neumann, Two combinatorial problems in group theory, Bull. Lond. Math. Soc. 21 (1989), 456-458.

[10] L. Ribes, P. Zalesskii, Profinite Groups, 2nd edition, Springer Verlag, Berlin, New York, 2010.

[11] D. J. S. Robinson, A course in the theory of groups, Second edition. Graduate Texts in Mathematics, 80. Springer-Verlag, New York, 1996.

[12] P. Rowley, Finite groups admitting a fixed-point-free automorphism group, J. Algebra, 174 (1995) 724-727.

[13] A. Shalev, Profinite groups with restricted centralizers. Proc. Amer. Math. Soc. 122 (1994), 1279-1284.

[14] J. Wiegold, Groups with boundedly finite classes of conjugate elements, Proc. Roy. Soc. London Ser. A 238 (1957), 389-401.

[15] J. S. Wilson, Profinite Groups, Clarendon Press, Oxford, 1998.

[16] E. I. Zelmanov, On periodic compact groups. Israel J. Math. 77, 83-95 (1992). 
Cristina Acciarri: Dipartimento di Scienze Fisiche, Informatiche e Matematiche, Università degli Studi di Modena e Reggio Emilia, Via Campi 213/B, I-41125 Modena, Italy

Email address: cristina.acciarri@unimore.it

Pavel Shumyatsky: Department of Mathematics, University of Brasilia, Brasilia-DF, 70910-900 BraZIL

Email address: pavel@unb.br 